\title{
An Inherited Cancer Syndrome Due to a Germline Monoallelic EGFR Mutation with Loss of Heterozygosity in Lung and Breast Tumors
}

\author{
Hanifeh Mirtavoos-Mahyari ${ }^{1 *}$, Farbod Bahreini ${ }^{2}$, Hassan Vahidnezhad ${ }^{3,4}$ \\ ${ }^{1}$ Lung Transplantation Research Center (LTRC), National Research Institute of Tuberculosis and \\ Lung Diseases (NRITLD), Shahid Beheshti University of Medical Sciences, Tehran, Iran \\ ${ }^{2}$ Department of Biochemistry, Faculty of Biological Sciences, Tarbiat Modares University, Tehran, \\ Iran \\ ${ }^{3}$ Department of Dermatology and Cutaneous Biology, Sidney Kimmel Medical College, Thomas \\ Jefferson University, Philadelphia, PA, USA \\ ${ }^{4}$ Jefferson Institute of Molecular Medicine, Thomas Jefferson University, Philadelphia, PA, USA
}

\section{* Corrresponding author:}

Hanifeh Mirtavoos-Mahyari M.D., Ph.D.

E-mail: hanifehmirmah@yahoo.com, Tel.: +98 (21) 27122002 Fax: +98 (21) 26109930

Address: National Research Institute of Tuberculosis and Lung Diseases (NRITLD), Daar-Abad, Niavaran, Tehran, Iran; Po Box: 19575-154 


\begin{abstract}
The epidermal growth factor receptor $(E G F R)$ exon-19 deletion is one of the most common mutations detected in lung cancer patients. Although exon-19 deletion is frequently detected in adenocarcinoma, observing this mutation in germline cells is very rare. Besides, the cooccurrence of homozygous and heterozygous mutations in dual primary cancers in a person is very uncommon. This article presents a 53-year-old Iranian woman with no history of smoking who was diagnose with two primary cancers; invasive ductal carcinoma, and primary pulmonary lung adenocarcinoma. The case reported a history of breast cancer in her sister and a history of lung cancer in her father. To select the best choice of treatment the EGFR gene was analyzed with Sanger's sequencing method from DNA extracted from the patient's lung tissue sample. Observing two primary cancers in this patient and considering her family pedigree, germline cells were also analyzed using samples recruited from the patient's peripheral blood to investigate any EGFR mutations in her germline cells. The obtained data revealed that the lung tissue of the patient carried a homozygous form of EGFR exon-19 deletion while her peripheral blood contained a heterozygous form of this mutation, which is exceptionally rare.
\end{abstract}

Keywords: Adenocarcinoma of lung; Ductal carcinoma; Breast Neoplasms; ErbB receptors; Germ cells 


\section{Introduction}

Epidermal growth factor receptor (EGFR) is a member of the ErbB family of receptor tyrosine kinases that applies its roles in the physiology of epithelial cells. Mutations in the EGFR coding gene are reported in different types of cancers including lung cancers. It is the target of several cancer therapies that are currently being adopted (e.g., Erlotinib and Gefitinib) [1]. Exon-19 deletions are the most common mutations in $E G F R$, which contribute to oncogenesis [2,3]. It is demonstrated that these mutations are correlated with sensitivity to inhibition of EGFR tyrosine kinase by the above-mentioned drugs [4]. Moreover, researches have shown that the exon-19 deletion of EGFR in non-small cell lung cancer patients is linked to an unusual pattern of brain metastasis [5]. It is well established that these mutations are heterozygous in the tumor tissues of patients, and identification of a disease-causing mutation with the homozygous form is extremely infrequent [6]. Besides, the co-occurrence of lung and breast cancers in the same person at the same time is indeed rare. Besides, data are extremely scarce about gene mutations that would predispose the incidence of two different cancers with different sources, simultaneously [7]. Herein, we report the effect of a germline EGFR mutation on the cooccurrence of lung and breast cancers in a 53-year-old patient with a family history of breast cancer in her sibling and a history of lung cancer in her parent. The result of this study can be used for clinical screening in patients who suffer lung cancer accompanied by breast cancer.

\section{Case Presentation, Methods, and Results}

A 53-year-old Iranian female presented with progressive dyspnea. The patient also complained of sharp upper back pain which was progressive, 10/10 in intensity, and alleviated with supine posture and analgesic medications. No significant motor or sensory symptoms, bladder or bowel incontinence were seen. She had been postmenopausal for 4 years with no history of alcohol intake, cigarette smoking, or oral contraceptive pill use. Figure 1A illustrates the family pedigree status of the patient. The proband (patient IV.1) was the oldest of four children. A 
history of breast cancer was raised in her sister (case IV.1) who died at age 34 and the proband's father (case III.1), who died at the age of 55, suffered from lung cancer.

On primary examination, the patient was cachectic, tachypneic (respiratory rate 24 times $/ \mathrm{min}$ ), and saturating index of oxygen was at $96 \%$ on ambient room air. Other vital signs were within normal range. On percussion of chest and auscultation breath, sounds were normal. There was a soft, non-tender, freely mobile mass in the upper outer quadrant of her right breast. No nipple or areolar discharge was detected. Supraclavicular and axillary lymph nodes were impalpable. Other Cardiovascular, abdominal, and neurological examinations were noncontributory.

A contrast-enhanced computed tomography (CT) of the thorax revealed a $41 \times 21 \mathrm{~mm}$ soft tissue density mass lesion involving the upper lobe of the left lung, mild bilateral pleural reactivation, nodular infiltration of the right breast, and lytic destructive lesions of left ribs (Figure 1B). Also, an irregular hypoechoic soft tissue density mass lesion measuring $2.5 \times 3.5$ $\times 3 \mathrm{~cm}$ was noted in the right breast in the mammographic exam. A whole-body bone scan showed numerous bone site metastasis. Histopathological examination of the left upper lobe mass biopsy (figure 1C, and 1D) showed features compatible with primary pulmonary lung adenocarcinoma (TTF-1 and Napsin A were both positive). Fine-needle aspiration of right breast mass (figure 1E) showed invasive ductal carcinoma (grade II). Thus, a diagnosis of synchronous dual primary cancers of the lung and breast was made. Based on clinical manifestations and the presence of a family history of cancer in this family, a hereditary form of cancer was considered for this family.

Accordingly, the polymerase chain reaction (PCR) and Sanger sequencing of EGFR of the DNA extracted from peripheral blood of the patients revealed a heterozygous in-frame mutation in exon-19 of EGFR: NM_005228.5: c.2237_2251del15, p.Glu746_Thr751delinsAla (Figure 1F). The mentioned exon of the EGFR gene was detected to be normal in the obtained 
blood samples from the mother and the brother of the proband (cases III.2 and IV.2); case IV.4 was unavailbe for a blood test at the time of experiment. The detected mutation in the proband is presented in the normal database of genetic variants in Gnomad in heterozygous and homozygous forms. Mutational analysis of DNA from lung tissue of the patients revealed the same mutation in EGFR but as a homozygous, further corroborating the existence of a hereditary form of cancer due to germline heterozygous EGFR mutation with extensive phenotypic heterogeneity in the family. The management of adenocarcinoma of the lung in this patient included palliative chemotherapy with $150 \mathrm{mg}$ per day of Erlotinib. After nine months of treatment, she developed lung metastases to the brain, and palliative brain radiotherapy (RT) was the choice for treatment.

\section{Discussion}

EGFR mutations not only promote lung adenocarcinoma but also are linked to the sensitivity to erlotinib and gefitinib. These mutations, when occurring in the kinase domain of EGFR, affect the molecular structure of this receptor, lead to abnormal activation of EGFR, and influence the downstream kinases, and cause anti-apoptotic and/or proliferation signaling [8]. EGFR exon-19 deletion, specifically, is reported to be responsible for the responsiveness to gefitinib in lung cancer [9]. It is demonstrated that exon-19 deletion (both germline and somatic mutations) elevates the autophosphorylation of EGFR, activates AKT and STAT pathways, and consequently promotes cell survival. In-silico analysis revealed that exon-19 deletion can shift the inactive form of EGFR to its active form [10]. This mutation can be detected in a homozygous or heterozygous manner [11,12]. Despite the ongoing research on this field, underlying two different cancers with different genotypes of germline mutations is rare. Herein, we reported a 53-year-old Iranian female who was diagnosed with dual primary cancers of lung and breast at the same time. Due to the family background of each of these cancers in her family, a hereditary form of cancer was considered for this family. A heterozygous mutation 
in exon-19 of EGFR (NM_005228.5: c.2237_2251del15,p.Glu746_Thr751delinsAla) of blood sample-extracted DNA was identified in the patient. On the other hand, analyzing lung tissueextracted DNA of the patient, the same mutation was detected but as a homozygous.

\section{Ethics Declaration}

The study design was approved by the ethics committee of Shahid Beheshti University of Medical Sciences. The Ethics committee approval number is IR.SBMU.NRITLD.REC.1399.187. Written informed consent was obtained from the patient.

\section{Data Availability Statement}

The datasets used and/or analyzed during the current study are available from the corresponding author on reasonable request.

\section{Author Contributions}

Hanifeh Mirtavoos-Mahyari defined the study protocol, contributed to the laboratory work, analyzed the data, and drafted the manuscript. Farbod Bahreini analyzed the data, provided the final revision of the manuscript, and was in charge of the submission process. Hassan Vahidnezhad analyzed the data and drafted the manuscript. All the authors have given the final approval of the version to be published and they take responsibility for appropriate portions of the content.

\section{Conflict of Interest}

The authors declare no potential conflict of interests.

\section{Funding}

The authors did not receive financial support for any part of this article. 


\section{Acknowledgments}

We would like to acknowledge the support of Shahid Beheshti University of Medical Sciences.

\section{References}

1. Sigismund, S.; Avanzato, D.; Lanzetti, L. Emerging functions of the EGFR in cancer. Mol Oncol 2018, 12, 3-20, doi:10.1002/1878-0261.12155.

2. Centeno, I.; Blay, P.; Santamaría, I.; Astudillo, A.; Pitiot, A.S.; Osorio, F.G.; González-Arriaga, P.; Iglesias, F.; Menéndez, P.; Tardón, A. Germ-line mutations in epidermal growth factor receptor (EGFR) are rare but may contribute to oncogenesis: a novel germ-line mutation in EGFR detected in a patient with lung adenocarcinoma. BMC cancer 2011, 11, 1-8.

3. Lou, Y.; Pecot, C.V.; Tran, H.T.; DeVito, V.J.; Tang, X.M.; Heymach, J.; Luthra, R.; Wistuba, I.I.; Zuo, Z.; Tsao, A.S. Case report: Germline mutation of T790M and dual/multiple EGFR mutations in patients with lung adenocarcinoma. Clinical lung cancer 2016, 17, e5.

4. Prudkin, L.; Tang, X.; Wistuba, I.I. Germ-line and somatic presentations of the EGFR T790M mutation in lung cancer. Journal of Thoracic Oncology 2009, 4, 139-141.

5. Sekine, A.; Kato, T.; Hagiwara, E.; Shinohara, T.; Komagata, T.; Iwasawa, T.; Satoh, H.; Tamura, K.; Kasamatsu, T.; Hayashihara, K. Metastatic brain tumors from non-small cell lung cancer with EGFR mutations: distinguishing influence of exon 19 deletion on radiographic features. Lung cancer 2012, 77, 64-69.

6. Lynch, T.J.; Bell, D.W.; Sordella, R.; Gurubhagavatula, S.; Okimoto, R.A.; Brannigan, B.W.; Harris, P.L.; Haserlat, S.M.; Supko, J.G.; Haluska, F.G. Activating mutations in the epidermal growth factor receptor underlying responsiveness of non-small-cell lung cancer to gefitinib. New England Journal of Medicine 2004, 350, 2129-2139.

7. Larouche, V.; Akirov, A.; Thain, E.; Kim, R.H.; Ezzat, S. Co-occurrence of breast cancer and neuroendocrine tumours: New genetic insights beyond Multiple Endocrine Neoplasia syndromes. Endocrinol Diabetes Metab 2019, 2, e00092, doi:10.1002/edm2.92.

8. Zhu, J.-q.; Zhong, W.-z.; Zhang, G.-c.; Li, R.; Zhang, X.-c.; Guo, A.-I.; Zhang, Y.-f.; An, S.-j.; Mok, T.S.; Wu, Y.-I. Better survival with EGFR exon 19 than exon 21 mutations in gefitinib-treated non-small cell lung cancer patients is due to differential inhibition of downstream signals. Cancer letters 2008, 265, 307-317.

9. Paez, J.G.; Janne, P.A.; Lee, J.C.; Tracy, S.; Greulich, H.; Gabriel, S.; Herman, P.; Kaye, F.J.; Lindeman, N.; Boggon, T.J.; et al. EGFR mutations in lung cancer: correlation with clinical response to gefitinib therapy. Science 2004, 304, 1497-1500, doi:10.1126/science.1099314.

10. Tamirat, M.Z.; Koivu, M.; Elenius, K.; Johnson, M.S. Structural characterization of EGFR exon 19 deletion mutation using molecular dynamics simulation. PLoS One 2019, 14, e0222814, doi:10.1371/journal.pone.0222814.

11. Nakano, H.; Soda, H.; Nakamura, Y.; Uchida, K.; Takasu, M.; Nakatomi, K.; Izumikawa, K.; Hayashi, T.; Nagayasu, T.; Tsukamoto, K.; et al. Different epidermal growth factor receptor gene mutations in a patient with 2 synchronous lung cancers. Clin Lung Cancer 2007, 8, 562 564, doi:10.3816/CLC.2007.n.043.

12. Schaller, A.; Beau-Faller, M.; Mennecier, B.; Renaud-Picard, B.; Weingertner, N.; Massard, G.; Quoix, E. Lung Adenocarcinoma with Pulmonary Miliary Metastases and Complex Somatic Heterozygous EGFR Mutation. Case Rep Oncol 2014, 7, 769-773, doi:10.1159/000369526. 


\section{Figure}

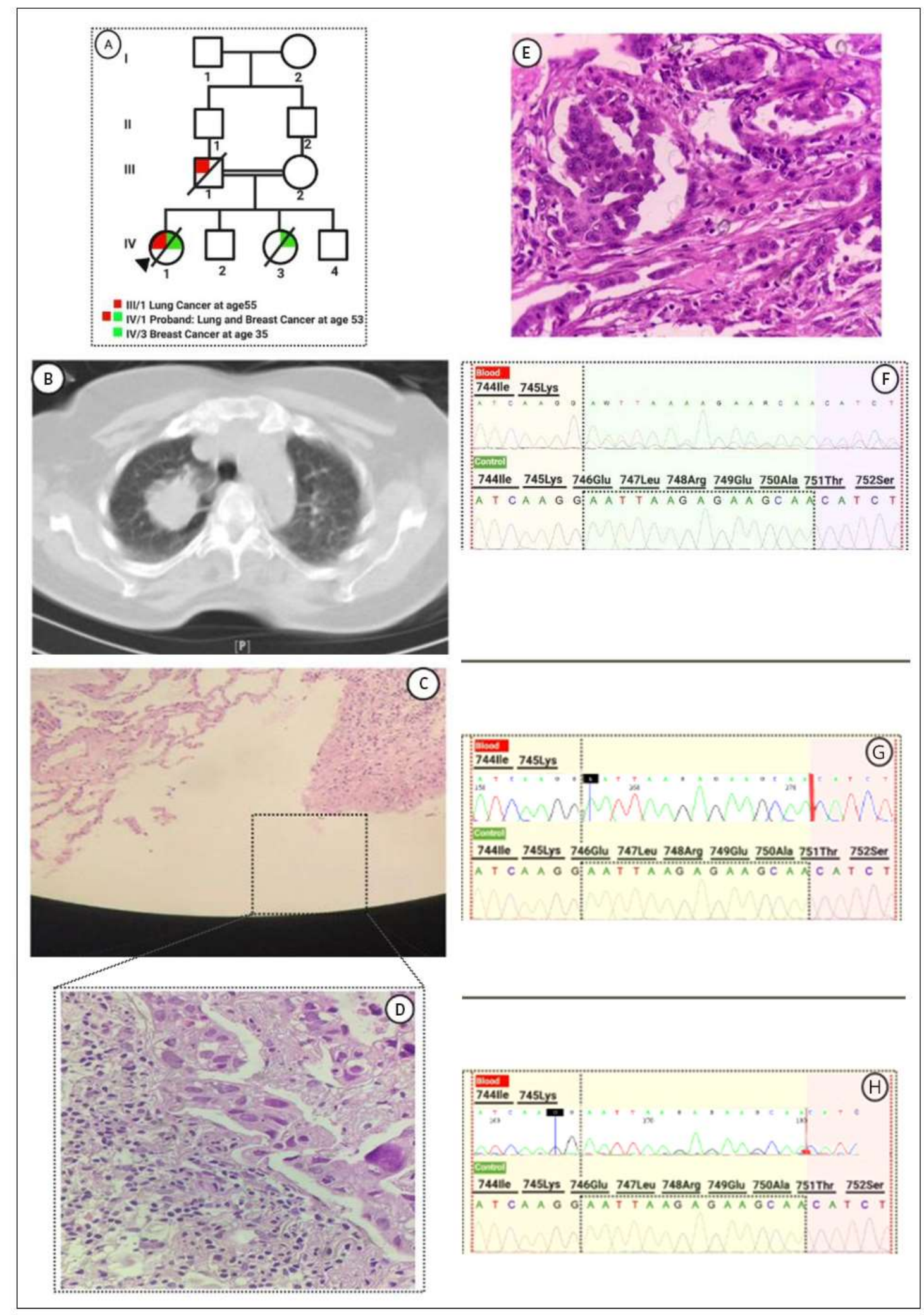


Figure 1. Identification of EGFR variant and results of clinical diagnostic procedures. Panel A: family pedigree status with respect to lung and breast cancers. Panel B: the contrast-enhanced CT scan of the thorax. Panels C and D: the histopathological examination result of the left upper lobe mass biopsy. Panel E: the result of fine-needle aspiration of the right breast mass. Panel F: sequence of the chromatogram of the blood genome, normal genome, and tumor genome in the patient. Panels G and H: sequence of the chromatogram of the blood genome and normal genome in the mother and brother of the proband. 